\title{
Fabrication and measurements of direct contact type RF MEMS switch
}

\author{
Jae-Hyoung Park ${ }^{\mathrm{a})}$ \\ Department of Physics, Ewha Womans University, \\ Jin Bldg. 335, 11-1 Daehyun-Dong, Seodaemun-gu, Seoul 120-750, Korea \\ a)parkjae@ewha.ac.kr
}

\begin{abstract}
This paper reports on the fabrication and measurements of a direct contact type RF MEMS switch. The switch is driven by the electrostatic force making the pass-through state with metal-to-metal direct contact. The actuation pad is connected with support beams to reduce switch deformation and increase contact force. The insertion loss and isolation of the switch have been measured and compared with the characteristics of the switch without support beams. The switch shows the isolation of $15.5 \mathrm{~dB}$ and the insertion loss of $0.34 \mathrm{~dB}$ at $50 \mathrm{GHz}$ with an applied DC bias of $35 \mathrm{~V}$. The switching time is measured to be $5.1 \mu \mathrm{s}$. Power handling capability and reliability of the fabricated switch have been discussed.
\end{abstract}

Keywords: RF MEMS switch, insertion loss, isolation, switching time, power handling capability, reliability

Classification: Micro- or nano-electromechanical systems

\section{References}

[1] H. Mizutani and Y. Takayama, "A DC-60 GHz GaAs MMIC switch using novel distributed FET," IEEE MTT-S Dig., pp. 439-442, 1997.

[2] S. P. Pacheco, L. P. B. Katehi, and C. T. Nguyen, "Design of low actuation voltage RF MEMS switch," IEEE MTT-S Dig., pp. 165-168, 2000.

[3] J. B. Muldavin and G. M. Rebeiz, "All metal high-isolation series and series/shunt MEMS switches," IEEE Microw. Wireless Compon. Lett., vol. 11, no. 9, pp. 373-375, 2001.

[4] Z. J. Yao, S. Chen, S. Eshelman, D. Denniston, and C. Goldsmith, "Micromachined low-loss microwave switches," Journal of Microelectromechanical Systems, vol. 8, no. 2, pp. 129-134, 1999.

[5] J. Oberhammer and G. Stemme, "Design and fabrication aspects of an S-shaped film actuator based DC to RF MEMS switch," Journal of Microelectromechanical Systems, vol. 13, no. 3, pp. 421-428, 2004.

[6] N. S. Barker and G. M. Rebeiz, "Optimization of distributed MEMS phase shifters," IEEE MTT-S Dig., pp. 299-302, 1999.

[7] M. Kim, J. B. Hacker, R. E. Mihailovich, and J. F. Denatale, "A monolithic MEMS switched dual-path power amplier," IEEE Microw. Wireless Compon. Lett., vol. 11, no. 7, pp. 285-286, 2001.

[8] D. Peroulis, S. Pacheco, K. Sarabandi, and L. P. B. Katehi, "Tunable 
lumped components with applications to reconfigurable MEMS filters," IEEE MTT-S Dig., pp. 341-344, 2001.

\section{Introduction}

Switching elements have been widely used in microwave and millimeter wave communication systems for signal routing in transmit and receive applications, switched line phase shifters in phased array antennas, and wide-band tuning networks. Usually, solid-state electronic devices using PIN diodes and GaAs MESFETs are integrated in the switching networks due to its integration compatibility and low manufacturing cost with batch fabrication [1]. These solid-state semiconductor switches show good performances at low frequency. However, the semiconductor switches have large insertion loss and poor isolation at the high frequency range over the $\mathrm{GHz}$ order. They are not suitable for the frequency agile wide band communication systems with a wide dynamic range due to the nonlinearity characteristics. RF MEMS switches, in which the electrical circuit elements are replaced with electromechanical signal processing components, were introduced as an alternative to these electrical switches $[2,3,4,5]$. Compared with the semiconductor switches, RF MEMS switches have low insertion loss, good isolation, low power consumption and good linearity. Therefore, there have been many efforts to commercialize RF MEMS switches in a number of applications such as front-end-modules (FEM), phase shifters, reconfigurable elements, and tunable filters $[6,7,8]$. Also, researches on integration of microelectromechanical switches with millimeter wave circuits have increased so as to improve the performances of devices.

In this paper, a direct contact type RF MEMS switch has been designed and fabricated to be applied to the miniaturized mobile communication systems and millimeter wave circuits. To reduce the beam deformation in the fabrication process and increase the contact force in the actuation of the switch, electroplated nickel structures are used as the structural material for the actuation pad and the actuation pad at both sides of the contact bar is connected with the support beams. The characteristics of the fabricated RF MEMS switch have been measured and discussed.

\section{Design}

The schematic view of the direct contact RF MEMS switch is shown in Fig. 1 (a). The switches are formed on the CPW transmission lines and actuated with electrostatic force. The width of the center signal line is $80 \mu \mathrm{m}$ and the gap between signal and ground line is $10 \mu \mathrm{m}$, having $50 \Omega$ characteristic impedance. The total length of the bridge type MEMS switch is $560 \mu \mathrm{m}$ and the width of actuation pad is $100 \mu \mathrm{m}$, respectively.

Figure 1(b) shows a SEM photograph of the fabricated switch. The contact bar of the switch is designed to suspend $1.5 \mu \mathrm{m}$ above the separated 


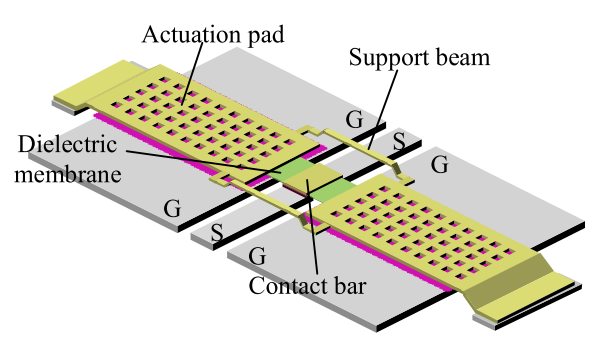

(a)

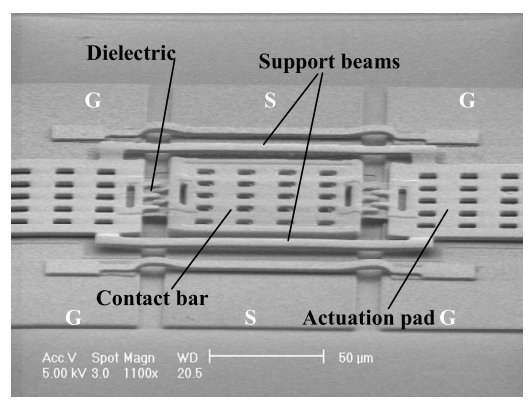

(b)

Fig. 1. Schematic view of direct contact type RF MEMS switch with support beams (a) and SEM photograph of the fabricated RF MEMS switch (b)

signal gap. The gap between the actuation pad and the bottom ground plate is $2 \mu \mathrm{m}$. When the DC voltage bias is applied between the actuation pad of the switch and the bottom ground plate, the switch is attracted down to the bottom plate by the electrostatic force. Because of this electrostatic actuation the contact electrode makes metal-to-metal electrical contact with the signal line crossing the separated signal line gap, resulting in the conduction state of the switch.

The contact bar and the actuation pad are connected through the insulating dielectric material to isolate the contact bar from the actuation section in the switch on state. Silicon nitride $\left(\mathrm{Si}_{3} \mathrm{~N}_{4}\right)$ film is used as an insulating material. The actuation pad at the both sides of the contact bar is connected with support beams, resulting in reduction of the beam deformation and the increase of contact force in the actuation of the switch. The fabrication yield is also improved by reducing the failure of the connecting insulator in the fabrication process. As shown in Fig. 1, the support beam is suspended higher than the contact bar. Therefore, the support beam does not degrade RF characteristics in the switching on and off states.

\section{Fabrication}

All the components of the switches are fabricated on a $520 \mu \mathrm{m}$-thick quartz substrate. The bottom CPW transmission lines are fabricated by electroplating of gold with the thickness of $2 \mu \mathrm{m}$. Electroplated nickel structures are used as the structural material for the actuation pad of the switches.

The fabrication process is as follows. First, $2-\mu \mathrm{m}$-thick gold transmission lines are electroplated. After formation of the CPW transmission lines, a dielectric layer is deposited on the limited CPW ground plate, which is beneath the driving electrode to avoid the possibility of a DC voltage short circuit. The gap between signal and ground line should be filled for planarization of the $2 \mu \mathrm{m}$-thick gold lines. As a gap filling material, photoresist is patterned and reflowed with curing. In this step, the sacrificial layer under the support beam is also defined for the support beam to be suspended higher than the contact bar and driving electrode. Therefore, the support beam does not affect RF characteristics in all the switching states. Photoresist is used as the 
sacrificial layer material. The sacrificial layer is patterned by UV lithography and thermally cured at a temperature of $210^{\circ} \mathrm{C}$. To increase the contact force in the actuation of the switch, the sacrificial layer on the contact part is slightly etched with anisotropic reactive ion etching, resulting in a smaller gap from the signal line compared with the gap between the actuation pad and ground plate. Next, the contact metal is electroplated with $1 \mu \mathrm{m}$-thick gold. Silicon nitride deposited using the plasma enhanced chemical vapor deposition (PECVD) process is patterned as an insulating layer connecting the contact bar and actuation pad. The $1.5 \mu \mathrm{m}$-thick nickel structures are electroplated as the actuation pad of the switches. Finally, the sacrificial layer is removed using the oxygen plasma process.

\section{Measurements and discussions}

\subsection{Insertion loss and isolation}

The responses of the fabricated switch were measured using an HP 8510XF network analyzer. The isolation of the switch represents the performance of the switch in the off state. The isolation is measured by S21 along the signal line with no applied voltage bias. The insertion loss of the switch is characterized by S21 through the input and output terminals in the on state of the switch. As shown in Fig. 1, the actuation pad at both sides of the contact bar is connected with support beams. With these support beams, the switch beam deformation is reduced and the contact force can be increased in the actuation of the switch. In Fig. 2, the measured insertion loss and isolation of the switch are shown and the results are compared with the characteristics of the switch without support beams. As shown in the results, the insertion loss is reduced with the support beam in the switch structure. The isolation in the switch off state was $15.5 \mathrm{~dB}$ and the insertion loss in the on state was measured $0.34 \mathrm{~dB}$ at $50 \mathrm{GHz}$ with an applied DC voltage bias of $35 \mathrm{~V}$.

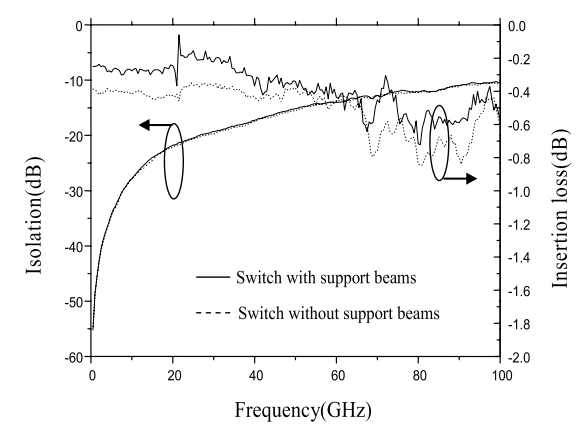

(a)

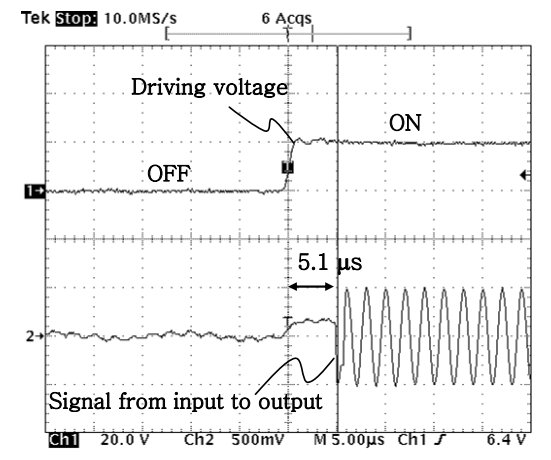

(b)

Fig. 2. Measured responses of the fabricated switch (a) insertion loss and isolation (b) switching time

The switch was fabricated on the $50 \Omega \mathrm{CPW}$ line and the length of the CPW line is $200 \mu \mathrm{m}$. The characteristics of the switch were measured through 
this CPW line. The insertion loss in the on state of the switch was measured $0.2 \mathrm{~dB}$ at $30 \mathrm{GHz}$. The measured contact resistance at this state is about $2 \Omega$. From the calculated electrostatic and restoring force of the switch in the contact state, the contact force can be calculated. The contact force at this operating voltage is calculated $80 \mu \mathrm{N}$, approximately. The isolation in the off state of the switch was about $20 \mathrm{~dB}$ at $30 \mathrm{GHz}$. The fitted up-state capacitance is about $6 \mathrm{fF}$. The spring constant and the resonant frequency of the switch have been simulated using ANSYS. The calculated spring constant is $18.7 \mathrm{~N} / \mathrm{m}$ and the resonant frequency is $32.9 \mathrm{kHz}$.

\subsection{Switching time}

The switching speed can be estimated by measuring the time taken by the switch operation to make the output signal reach the steady state from the point that the control driving voltage is applied. From these definitions, the switching time of the fabricated MEMS switch was tested. Figure 2 (b) shows the measured switching time. The switching time is measured to be $5.1 \mu \mathrm{s}$ when the switch is turned on. The measured turn-off switching time is $1.1 \mu \mathrm{s}$. The switching time is determined by the resonant frequency of the switch and damping effect. In the switch operations, damping effect is dominant factor to slow down the switching speed. Increase of the actuation pad size makes the damping effect more serious, resulting in longer switching time. The fabricated switch with the actuation pad width of $160 \mu \mathrm{m}$ shows the switching time of $13 \mu \mathrm{s}$.

\subsection{Power handling capability}

Power handling capability of the RF MEMS switch is limited due to the self actuation and stiction in the down state at high incident RF power. In this paper, the power handling capability of the switch has been measured. Figure 3 shows the schematic measurement setup and the self actuation test results. The switch is initially in the up state without DC bias and the input $\mathrm{RF}$ power is slowly increased up to a maximum of $35 \mathrm{dBm}(2.5 \mathrm{~W})$ at $2 \mathrm{GHz}$. As shown in Fig. 3, the switch is actuated with no bias voltage, when the incident $\mathrm{RF}$ power is higher than $1 \mathrm{~W}$. This result shows that the induced

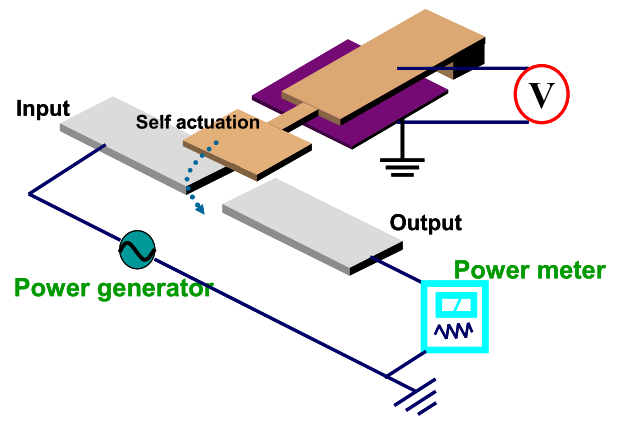

(a)

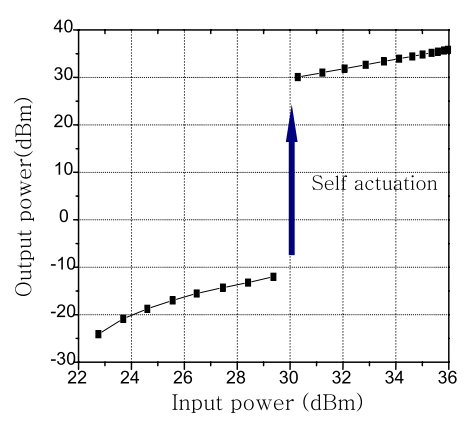

(b)

Fig. 3. Schematic measurement setup for power handling capability (a) and self actuation test results (b) 
force from the input RF signal can cause unintended switch actuation without any DC bias voltage.

Welding problem in the switch on state is also the main cause of the switch failure in the high power applications. The RF power generating welding phenomenon is measured by detecting the power at which on and off switching actuation fails during hot switching conditions. The switch is actuated with DC bias and maintained in down state for 10 minutes at each input power. The RF MEMS switch presented in this paper showed welding phenomenon at the input power of $0.5 \mathrm{~W}$. For the switch with high power handling capability, it is necessary to develop not only contact materials but also the actuator design to increase contact force and restoring force.

\subsection{Lifetime of the switch}

The Au-to-Au contact reliability of the fabricated RF switch has been tested by cycling the switch actuation. The frequency of the switch actuation is $1 \mathrm{kHz}$. When cold-switched, significant changes in contact resistance were not observed until $10^{7}$ times repeated actuations. However, after approximately $10^{7}$ cycles, the contact resistance was drastically increased by about $2 \Omega$ and finally stiction occurred. In cold switching conditions, no arcing occurs across the contacts. Failure is due to the erosion and wears in the contact surfaces. When the fabricated device was hot switched with the conduction current of $50 \mathrm{~mA}$, the failure was observed after $10^{5}$ cycles of actuation. It is known that resistance change and failure at hot switching are influenced by complex factors like hardness, oxidation, organic contamination, and melting point of contact material. High current density on the contact surface accelerates wear progress and material transfer at constriction contact.

\section{Conclusion}

In this paper, a direct contact type RF MEMS switch has been designed, fabricated and tested. The support beams are employed to reduce beam deformation in the fabrication process and to increase contact force in the actuation of the switch. The total length of the switch is $560 \mu \mathrm{m}$ and the width is $100 \mu \mathrm{m}$, respectively. The measured insertion loss of the switch is as low as $0.34 \mathrm{~dB}$ at $50 \mathrm{GHz}$ with the applied voltage bias of $35 \mathrm{~V}$. The isolation of the switch is $15.5 \mathrm{~dB}$ at $50 \mathrm{GHz}$. The switching time is measured to be $5.1 \mu \mathrm{s}$. Power handling capability of the switch has been measured with the increase of incident $\mathrm{RF}$ power at $2 \mathrm{GHz}$. Self actuation was observed when the input RF power is higher than $1 \mathrm{~W}$ and the welding phenomenon occurred at the RF power of $0.5 \mathrm{~W}$. When cold switched, the switch shows $10^{7}$ cycles reliability, while the failure occurs after $10^{5}$ times repeated actuations in hot switching conditions with the conduction current of $50 \mathrm{~mA}$. These results show that the RF MEMS switch proposed in this paper has the feasibility to be applied to the miniaturized mobile communication systems and millimeter wave circuits. 


\section{Acknowledgments}

This work was supported by Creative Research Initiatives (MEMS Space Telescope) of MOST/KOSEF. 